\title{
Influência de gênero, idade e renda sobre o bem-estar de idosos cuidadores e não cuidadores
}

\author{
Influence of gender, age and income on the well-being of elderly caregivers
}

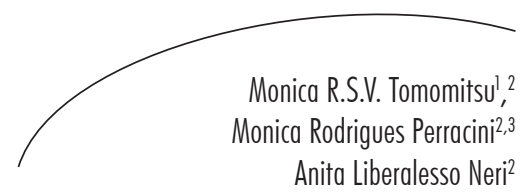

\section{Resumo}

Objetivos: Comparar idosos cuidadores e não cuidadores quanto às condições de saúde, psicossociais e de desempenho funcional e conhecer a influência das variáveis socioeconômicas sobre estes grupos. Método: Estudo envolvendo idosos residentes de três cidades brasileiras - Parnaíba-PI, Campinas-SP e Ivoti-RS - com base nos dados do estudo FIBRA. A amostra foi composta de 676 idosos, 338 cuidadores e 338 não cuidadores. Os cuidadores responderam afirmativamente a duas questões sobre a prestação de cuidado no Elders Life Stress Inventory. Os instrumentos utilizados foram: versões brasileiras da Geriatric Depression Scale, da Interpersonal Support Evaluation List, da escala de atividades instrumentais de vida diária (AIVD), de 13 itens de atividades avançadas de vida diária e escala de satisfação com a vida. Foram aplicadas questões de autorrelato sobre idade, gênero, renda familiar, número de doenças e fadiga. Resultados: Os cuidadores compuseram $29,7 \%$ da amostra. A tarefa de cuidar era mais frequente entre as mulheres $(65,7 \%)$, os idosos de 65 a 74 anos e os de menor renda familiar. Houve frequência maior de queixa de insônia entre os cuidadores comparados com os não cuidadores $(\mathrm{p}=0,013)$. Para os grupos, ser mais pobre significou maior frequência de insônia e de dependência em AIVD, além de mais sintomas depressivos entre os cuidadores $(p=0,24)$. As mulheres eram as mais doentes e os homens os mais isolados socialmente. Os cuidadores mais velhos reportaram maior grau de dependência em AIVD ( $\mathrm{p}=0,015)$. Conclusão: Circunstâncias socioeconômicas podem contribuir para desfechos negativos sobre as condições de saúde física e bem-estar psicológico, especialmente entre os cuidadores.

\footnotetext{
Faculdade de Ciências Médicas. Comissão de Pós-graduação. Universidade Estadual de Campinas. Campinas, SP, Brasil.

2 Faculdade de Ciências Médicas. Universidade Estadual de Campinas. Campinas, SP, Brasil.

Programa de Mestrado e Doutorado em Fisioterapia. Universidade Cidade de São Paulo. São Paulo, SP, Brasil.

Universidade Estadual de Campinas - Unicamp, Faculdade de Ciências Médicas, Comissão de Pós-graduação Av. Tessália Vieira de Camargo, 126, Cidade Universitária, Distrito de Barão Geraldo

13083-970, Campinas, SP, Brazil.

E-mail: monicatomomitsu@gmail.com
}

Palavras-chave: Cuidadores Saúde do Idoso. Depressão. Fatores Socioeconômicos. Identidade de Gênero. Envelhecimento. 


\section{Abstract}

Objectives: To compare elderly caregivers with non-caregivers regarding their health psychosocial and functional status, and to investigate the influence of socioeconomic variables on these conditions in both groups. Methods: A study involving elderly residents from three Brazilian cities - Parnaíba-PI, Campinas-SP and Ivoti-RS - from FIBRA study. The sample was composed of 676 older adults, 338 caregivers and 338 noncaregivers. Caregivers were identified through two questions of the Elders Life Stress Inventory (ELSI) regarding care provision. The instruments used were: the Brazilian versions of the Geriatric Depression Scale, the Interpersonal Support Evaluation List and the Lawton instrumental Activities of Daily Living Scale (IADL), and 13 items of advanced activities of daily living and a scale of life satisfaction. We applied self-report questions about age, gender, family income, number of diseases and fatigue. Results: The elderly caregivers were about $29 \%$ of the sample. The task of caring was more frequent among women (65.7\%), aged between 65 and 74 years and among those with lower income. A higher frequency of insomnia complaint was observed among caregivers compared to non-caregivers $(\mathrm{p}=0.013)$. For both groups, low income influenced the quality of sleep and dependence in IADL and more depressive symptoms among caregivers $(p=0.24)$. In addition women had a greater number of diseases and men were more restricted in social participation. Older caregivers were more dependent in IADL $(p=0.015)$. Conclusion: Socioeconomic status can influence negatively in physical health status and psychological well-being, particularly among elderly caregivers.
Key words: Caregivers. Health of the Elderly. Depression. Socioeconomic Factors. Gender Identity. Aging.

\section{INTRODUÇÃO}

A obrigatoriedade moral de prestar cuidados a idosos física e cognitivamente doentes e incapacitados é registrada em códigos legais e religiosos, que se baseiam nos princípios da reciprocidade e da solidariedade para exigir que a família e outras instituições sociais respondam pelo bem-estar dos mais velhos. Em todo o mundo, a família é reconhecida como o locus privilegiado da prestação de cuidados materiais, instrumentais e emocionais aos idosos que deles necessitam. $\mathrm{Na}$ atualidade, é crescente a probabilidade de que idosos sejam cuidados por outros idosos, porque tanto os que precisam de ajuda quanto seus cuidadores potenciais estão vivendo mais tempo.

O aparecimento ou o agravamento de doenças crônicas e incapacidades decorrentes do avanço da idade associa-se ao aumento da susceptibilidade ao ônus físico e psicológico gerado pela prestação de cuidados a outros idosos. ${ }^{2}$ Cuidadores idosos têm mais problemas de saúde, ${ }^{3}$ mais depressão e menor satisfação com a vida ${ }^{4,5}$ e, além disso, recebem menos apoio social do que idosos não cuidadores. ${ }^{6}$ De acordo com dados da metaanálise conduzida por Pinquart \& Sörensen, cuidadores idosos que recebem pouco apoio social tendem a apresentar níveis mais baixos de saúde subjetiva, mais sintomas depressivos e maior procura por serviço de saúde do que cuidadores que são adultos jovens e de meia-idade. Em contrapartida, cuidadores idosos podem ser mais afetados pelo ônus físico do cuidado do que cuidadores de meia-idade, mas, graças à presença de estratégias eficazes de enfrentamento, podem apresentar níveis mais baixos de estresse e avaliações mais positivas de suas habilidades para cuidar do que estes últimos. ${ }^{8}$ Nesse sentido, Smith et al. ${ }^{9}$ observaram que cuidadores de meiaidade (40 a 59 anos) requeriam acompanhamento mais frequente e mais suporte informativo e educacional proveniente de profissionais da saúde do que cuidadores idosos. Deste intrincado conjunto de fatos decorrem novos e importantes desafios para os mais velhos, para suas famílias e para o sistema social.

Cumprindo atribuições de gênero, as mulheres são os cuidadores familiares mais prováveis. Mulheres cuidadoras são geralmente esposas 
ou filhas dos idosos que são alvos do cuidado. Uma questão demográfica e epidemiológica importante que se coloca para as famílias e para as sociedades é que as mulheres idosas tendem a ter mais doenças crônicas, incapacidades e sintomas depressivos, assim como tendem a exibir níveis mais baixos de satisfação com a vida do que os homens idosos. ${ }^{4}$ Ou seja, as desvantagens do declínio da saúde associado ao avanço da idade e ao gênero feminino podem interagir com o ônus físico e psicológico da prestação de cuidado, prejudicando a qualidade de vida objetiva e subjetiva das mulheres idosas cuidadoras. Níveis mais altos de estresse e maior susceptibilidade aos efeitos deletérios da prestação de cuidados foram observados em mulheres em comparação com homens, particularmente na presença de apoio social insuficiente. ${ }^{4}$ Foi observada uma associação substancial entre o estresse relacionado a prestar cuidados, maior número de doenças crônicas e de sintomas depressivos em cuidadores de idosos com demência e em homens cuidadores. ${ }^{10}$ No entanto, os efeitos da prestação de cuidados sobre a saúde se diluem quando a variável gênero é controlada.

De acordo com o modelo do estresse do cuidador desenvolvido por Pearlin et al., ${ }^{11}$ o bem-estar físico e psicológico dos cuidadores pode ser afetado não só pelo ônus gerado pelas tarefas de cuidado, o qual pode ser intensificado por condições de saúde associadas à idade e ao gênero, como também por variáveis demográficas e socioeconômicas que determinam o grau de acesso da família a recursos sociais e materiais. Pinquart \& Sörensen ${ }^{5}$ encontraram correlação negativa entre saúde física e idade e correlação positiva entre status socioeconômico e suporte informal em cuidadores familiares. Borg \&

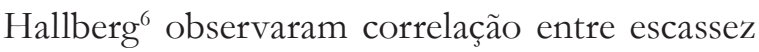
de recursos financeiros e baixa satisfação com a vida, particularmente entre cuidadores idosos com baixo nível de suporte social percebido.

Pesquisas enfocando idosos cuidadores vivendo em diferentes contextos socioeconômicos ainda não foram suficientemente exploradas, principalmente na população brasileira. Esse conhecimento pode contribuir de forma importante para a elaboração de políticas públicas e para a análise da demanda por serviços formais de cuidado e de proteção a idosos. Conhecer as diferenças entre condições de saúde e de funcionalidade de idosos cuidadores e não cuidadores é fundamental para se estabelecer protocolos de avaliação e de acompanhamento realizados por profissionais de saúde de modo a favorecer a qualidade de vida tanto dos idosos que prestam cuidados quanto dos que são cuidados por estes.

Os objetivos deste estudo foram: comparar idosos cuidadores com não cuidadores quanto às variáveis "número de doenças", "fadiga", "sintomas depressivos", "insônia", "satisfação com a vida", "atividades instrumentais de vida diária (AIVD)", "atividades avançadas de vida diária (AAVD)" e "suporte social percebido", e conhecer a influência das variáveis sociodemográficas e econômicas sobre estas variáveis de interesse.

\section{MATERIAIS E MÉTODOS}

Este estudo envolveu idosos residentes em três cidades brasileiras, Parnaíba-PI, CampinasSP e Ivoti-RS, cujos dados compunham o banco eletrônico de um estudo que integra os trabalhos de uma rede de pesquisa conhecida como FIBRA (acrônimo de rede de Pesquisa sobre Fragilidade em Idosos Brasileiros) sobre relações entre fragilidade e condições sociodemográficas, psicossociais e de saúde, cognição e funcionalidade.

Em cada cidade, a seleção dos idosos foi feita por amostragem aleatória simples dos setores censitários urbanos, em cujos domicílios foram recrutadas cotas de homens e mulheres que neles tinham residência permanente, os quais tinham idade entre 65 e 69, 70 e 74, 75 e 79 anos e de 80 anos e mais. As cotas eram proporcionais à presença dos grupos de idade e sexo na população urbana. Por ocasião do recrutamento, foram incluídos idosos sem limitações graves de natureza física, sensorial, cognitiva, de mobilidade ou de comunicação. 
Nas três localidades, o recrutamento e a coleta de dados foram feitos em ondas sucessivas, no período de 2008-2009. Em cada uma, os idosos eram recrutados em número que satisfizesse os limites preestabelecidos pelo plano amostral e encaminhados para a coleta de dados. Cada onda de recrutamento e de coleta de dados era encerrada quando o contingente de idosos esperados havia comparecido e participado efetivamente da pesquisa. As três cidades foram selecionadas dentre as sete do estudo maior porque seus participantes responderam aos itens sobre cuidado de interesse para esta investigação. Detalhes metodológicos sobre o estudo principal e sobre as características sociodemográficas das amostras podem ser encontrados em Neri et al. ${ }^{12}$

O estudo foi conduzido segundo as normas da última Declaração de Helsinki para pesquisa com seres humanos e foi aprovado pelo Comitê de Ética em Pesquisa da Faculdade de Ciências Médicas (CEP/FCM) mediante os pareceres $n$. 208/2007 e 068/2011. Todos os participantes assinaram o Termo de Consentimento Livre e Esclarecido.

\section{Participantes}

Dentre os 1.581 idosos que compunham as amostras das três cidades, 442 foram excluídos no início da coleta de dados em razão de terem pontuado abaixo da nota de corte no Miniexame do Estado Mental, considerando-se o nível de escolaridade..$^{13}$ Os 1.139 idosos remanescentes foram considerados para a seleção do grupo de cuidadores. A condição de cuidador foi evidenciada por resposta afirmativa a dois itens do The Elders Life Stress Inventory (ELSI) ${ }^{14}$ que perguntavam se haviam cuidado do cônjuge ou de pais ou sogros idosos nos últimos cinco anos. Um total de 338 participantes cumpriu esta condição de cuidado. Dentre os 801 idosos não cuidadores foram sorteados 338, em cotas comparáveis às dos idosos cuidadores, considerando-se as variáveis: cidade de residência, gênero, idade e nível de renda familiar. Desta forma, integraram a amostra 676 idosos, dentre os quais 338 cuidadores e 338 não cuidadores.
Instrumentos

As variáveis de interesse para este projeto foram investigadas mediante as condições que se seguem.

1) Idade, gênero e renda familiar: três questões de autorrelato, cujos resultados eram anotados pelos avaliadores no formulário de pesquisa (data de nascimento, gênero masculino $\mathrm{x}$ feminino e renda familiar em valores brutos). Os valores de renda foram agrupados em cinco faixas de salários mínimos $(\mathrm{SM}):<1$; 1,1 a $3 ; 3,1$ a $5 ; 5,1$ a 10, e $>10$ SM. As idades foram agrupadas em quatro faixas: 65-69; 70 74; 75-79 e 80 e + .

2) Número de doenças: avaliado por meio de nove itens dicotômicos que investigavam se algum médico havia dito que o participante tinha as seguintes doenças crônicas: doença do coração; hipertensão; AVC/isquemia/ derrame; diabetes mellitus; câncer; artrite ou reumatismo; doenças dos pulmões; depressão e osteoporose. As respostas afirmativas foram contadas e foram criadas três categorias: zero, 1 ou 2 e 3 ou mais doenças.

3) Fadiga: avaliada por dois itens escalares de autorrelato extraídos da Center for Epidemiological Studies-Depression (CES-D). ${ }^{15}$ Foram considerados com fadiga os idosos que responderam "sempre" ou "na maioria das vezes" a qualquer um ou a ambos os itens.

4) Sintomas depressivos: avaliado por meio da Escala de Depressão Geriátrica (EDG) ${ }^{16} \mathrm{com} 15$ itens com nota de corte maior do que 6 pontos. $\mathrm{O}$ coeficiente de confiabilidade interna (alfa de Cronbach) da EDG na amostra deste estudo foi 0,734 .

5) Insônia: avaliada por quatro questões de autorrelato, que comportavam respostas "sim" ou "não", extraídas do Perfil de Saúde de Nottingham (PSN). ${ }^{17}$ Foram considerados com sintomas de insônia aqueles que responderam positivamente a qualquer um dos quatro itens. 
6) Satisfação com a vida: foi avaliada mediante um questionário extraído da literatura, ${ }^{18,19}$ com um item sobre a satisfação global hoje, um item sobre a satisfação em comparação com os outros da mesma idade e seis itens sobre a satisfação referenciada aos domínios como "memória", "capacidade de fazer e solucionar coisas", "amizades e relações familiares", "ambiente", "acesso a serviços de saúde e meios de transporte". Eram respostas escalares de 3 pontos, podendo a pontuação variar de 1 a 24 . A mediana foi calculada e usada como ponto de corte $(=21)$. Aqueles que pontuaram $<21$ foram considerados com menor satisfação com a vida e os que pontuaram $>22$ pontos com maior satisfação com a vida. O alfa de Cronbach foi de 0,729.

7) Atividades avançadas de vida diária (AAVD): consideradas como indicador de participação social, foram avaliadas mediante um questionário construído com base na literatura ${ }^{19,20}$ contendo 13 itens de autorrelato. Os itens incluíam: fazer e receber visitas, ir à igreja, ir a festas e a eventos culturais, realizar dança de salão, fazer viagens curtas e longas, dirigir automóvel, desempenhar trabalho remunerado e voluntário, participar de universidade da terceira idade e de centro de convivência, e participar de diretorias ou conselhos de sociedades. Para cada um dos itens havia três alternativas de resposta: nunca fiz, ainda faço e parei de fazer. Foram desconsideradas as atividades que nunca havia realizado e contada a frequência das que parou de fazer. Neste estudo, o número de AAVDs abandonadas foi considerada como indicador de participação social. Para tanto, foram criadas três faixas: $<2,2$ ou $3,>3$.

8) Atividades instrumentais de vida diária (AIVD): sete itens de autorrelato baseados na escala de Lawton \& Brody ${ }^{21}$ que avaliaram o nível de independência para AIVD. Havia três possibilidades de respostas: "totalmente independente"; "precisa de alguma ajuda"; e "precisa de ajuda total" para fazer as coisas do dia a dia. As respostas foram agrupadas em duas categorias. Na primeira, chamada de independência para AIVD, foram computados os itens em que os idosos responderam não precisar de nenhuma ajuda instrumental. $\mathrm{Na}$ segunda, foram computados os itens em que os idosos disseram que precisavam de ajuda parcial. Não foram registradas respostas indicativas de necessidade de ajuda total nesse instrumento.

9) Suporte social percebido: foram aplicados cinco itens semanticamente adaptados para o português do inventário Interpersonal Support Evaluation List (ISEL), ${ }^{22}$ cada um com quatro intensidades. A pontuação varia de 1 a 20. Foram calculados os tercis relativos aos escores da amostra. Aqueles que pontuaram <16 foram considerados com baixo suporte social percebido; entre 17 e 19 pontos, com médio suporte social percebido; e 20 pontos, com alto suporte social percebido. O coeficiente de confiabilidade interna foi 0,756 .

10) Intensidade do estresse associado à prestação de cuidados: foi avaliada por meio de item único de natureza escalar (0-5 pontos, em que 1 correspondia a menor estresse), ${ }^{14}$ aplicada apenas aos idosos que haviam respondido afirmativamente ao item de cuidado, que perguntava qual tinha sido a intensidade do estresse vivenciado na situação.

\section{Análise de dados}

Foi efetuado o teste Kolmogorov-Smirnov para aferir a normalidade da amostra, o qual revelou inexistência de distribuição normal. Foram adotados testes não paramétricos para as análises descritivas: qui-quadrado ou exato de Fisher para a comparação entre as frequências das variáveis categóricas; Mann-Whitney e Kruskal-Wallis para a comparação das médias das amostras independentes de cuidadores e não cuidadores nas variáveis de interesse, conforme os critérios de sexo, idade e nível de renda. O nível de significância adotado correspondeu a $\mathrm{p} \leq 0,05$. 


\section{RESULTADOS}

Dentre os 1.139 idosos com 65 anos e mais selecionados, 29,7\% estiveram envolvidos em cuidados ( $\mathrm{n}=338)$. Não foram encontradas diferenças significativas entre as frequências percentuais deidosos cuidadores e não cuidadores, conforme as variáveis "cidade", "sexo", "idade" e "renda familiar". Na composição dos grupos, a maioria dos idosos eram mulheres $(65,8 \%)$, com idade variando de 65 a 90 anos e média (dp) de $71,5( \pm 4,8)$ anos. A renda familiar média (dp) era

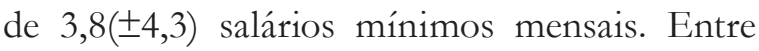
os cuidadores, mais mulheres do que homens estavam envolvidos com os cuidados, $65,7 \%$ contra 34,3\%. A maioria dos idosos cuidadores prestava cuidados aos cônjuges (76,3\%), 15,7\% aos pais e $7,7 \%$ tanto a cônjuge quanto a pais. A média (dp) da intensidade de estresse gerado pela experiência do cuidado foi de $3,8( \pm 1,3)$ pontos.

Dentre os idosos cuidadores do gênero feminino, a idade média (dp) foi de $71( \pm 4,8)$, enquanto entre os homens foi de $72,2( \pm 4,7)$ $(\mathrm{p}=0,011)$. Para os idosos não cuidadores, a idade média (dp) das mulheres foi de $71,1( \pm 4,8)$ e os homens foi de $72,3( \pm 4,6)(\mathrm{p}=0,015)$.

A tabela 1 oferece dados de comparação entre os grupos de cuidadores e não cuidadores quanto às variáveis sociodemográficas, de saúde, de atividades de vida diária, de satisfação com a vida e suporte social. Os cuidadores apresentaram frequência significativamente maior de insônia do que não cuidadores. Não foram observadas outras diferenças entre as frequências percentuais de cuidadores e não cuidadores nas demais variáveis consideradas.

Tabela 1 - Análise descritiva das variáveis da amostra em cuidadores e não cuidadores (n=676), FIBRA. Campinas-SP, 2008-2009.

\begin{tabular}{|c|c|c|c|}
\hline Variáveis & $\begin{array}{c}\text { Cuidadores } \\
\quad(\mathrm{n}=338)\end{array}$ & $\begin{array}{c}\text { Não cuidadores } \\
\quad(\mathrm{n}=338)\end{array}$ & $\mathrm{p}$ \\
\hline Gênero & & & 1,000 \\
\hline Feminino & $222(65,7 \%)$ & $222(65,7 \%)$ & \\
\hline Masculino & $116(34,3 \%)$ & $116(34,3 \%)$ & \\
\hline Faixa etária & & & 1,000 \\
\hline $65-69$ & $133(39,3 \%)$ & $133(39,3 \%)$ & \\
\hline $70-74$ & $127(37,6 \%)$ & $127(37,6 \%)$ & \\
\hline $75-79$ & $55(16,3 \%)$ & $55(16,3 \%)$ & \\
\hline 80 ou mais & $23(6,8 \%)$ & $23(6,8 \%)$ & \\
\hline Renda familiar & & & 0,966 \\
\hline$\leq 1,0 \mathrm{SM}$ & $25(7,4 \%)$ & $26(7,7 \%)$ & \\
\hline $1,1-3,0 \mathrm{SM}$ & $153(45,3 \%)$ & $156(46,2 \%)$ & \\
\hline $3,1-5,0 \mathrm{SM}$ & $67(19,8 \%)$ & $64(18,9 \%)$ & \\
\hline $5,1-10,0 \mathrm{SM}$ & $41(12,1 \%)$ & $41(12,1 \%)$ & \\
\hline$>10,0 \mathrm{SM}$ & $19(5,6 \%)$ & $15(4,5 \%)$ & \\
\hline Não responderam & $33(9,8 \%)$ & $36(10,6 \%)$ & \\
\hline Número de doenças & & & 0,554 \\
\hline Zero & $40(11,8 \%)$ & $36(10,7 \%)$ & \\
\hline 1 ou 2 & $152(45,0 \%)$ & $166(49,1 \%)$ & \\
\hline 3 ou mais & $146(43,2 \%)$ & $136(40,2 \%)$ & \\
\hline
\end{tabular}




\begin{tabular}{|c|c|c|c|}
\hline Variáveis & $\begin{array}{c}\text { Cuidadores } \\
\quad(\mathrm{n}=338)\end{array}$ & $\begin{array}{c}\text { Não cuidadores } \\
\quad(\mathrm{n}=338)\end{array}$ & $\mathrm{p}$ \\
\hline Fadiga & & & 0,167 \\
\hline Não & $249(73,7 \%)$ & $264(78,1 \%)$ & \\
\hline $\operatorname{Sim}$ & $84(24,8 \%)$ & $69(20,4 \%)$ & \\
\hline Não responderam & $5(1,5 \%)$ & $5(1,5 \%)$ & \\
\hline Insônia & & & $0,013^{\mathrm{a}}$ \\
\hline Não & $135(39,9 \%)$ & $\underline{167(49,4 \%)}$ & \\
\hline $\operatorname{Sim}$ & $\underline{203(60,1 \%)}$ & $171(50,5 \%)$ & \\
\hline Sintomas depressivos & & & 0,863 \\
\hline Não (GDS< <5) & $270(79,9 \%)$ & $269(79,6 \%)$ & \\
\hline $\operatorname{Sim}(G D S>0)$ & $67(19,8 \%)$ & $69(20,4 \%)$ & \\
\hline Não responderam & $1(0,3 \%)$ & - & \\
\hline Satisfação com a vida & & & 0,189 \\
\hline Menor $(<21)$ & $186(55,0 \%)$ & $166(49,1 \%)$ & \\
\hline Maior (>22) & $146(43,2 \%)$ & $160(47,3 \%)$ & \\
\hline Não responderam & $6(01,8 \%)$ & $12(3,6 \%)$ & \\
\hline AAVDs abandonadas & & & 0,084 \\
\hline$<2$ & $71(21,0 \%)$ & $95(28,1 \%)$ & \\
\hline $2-3$ & $164(48,5 \%)$ & $143(42,3 \%)$ & \\
\hline$>3$ & $103(30,5 \%)$ & $100(29,6 \%)$ & \\
\hline AIVDs & & & 0,051 \\
\hline Independente $(>7)$ & $196(58,0 \%)$ & $220(65,1 \%)$ & \\
\hline Dependência parcial & $142(42,0 \%)$ & $117(34,6 \%)$ & \\
\hline Não responderam & - & $1(0,3 \%)$ & \\
\hline Suporte social & & & 0,518 \\
\hline Baixo $(<16)$ & $108(32,0 \%)$ & $115(34,0 \%)$ & \\
\hline Médio (17-19) & $86(25,4 \%)$ & $94(27,8 \%)$ & \\
\hline Alto $(\geq 20)$ & $139(41,1 \%)$ & $125(37,0 \%)$ & \\
\hline Não responderam & $5(1,5 \%)$ & $4(1,2 \%)$ & \\
\hline
\end{tabular}

${ }^{a}$ Sign para $\mathrm{p}=0,013(\chi 2=6,13 ; \mathrm{GL}=1) . \mathrm{SM}=$ Salário Mínimo; GDS: Geriatric Depression Scale; AAVDs=Atividades Avançadas de Vida Diária; AIVDs=Atividades Instrumentais de Vida Diária.

No grupo de cuidadores, as mulheres apresentaram frequência significativamente maior de três ou mais doenças quando comparadas aos homens. Em contrapartida, houve proporção significativamente mais alta de homens que relataram ter abandonado um número maior de atividades avançadas de vida diária do que as mulheres. Estes resultados se repetiram para o grupo de não cuidadores. Adicionalmente, verificou-se que as mulheres não cuidadoras relataram frequência significativamente maior de fadiga do que os homens não cuidadores (tabela 2). 
Tabela 2 - Comparação das variáveis dependentes entre gêneros de cuidadores ( $\mathrm{n}=338$ ) e não cuidadores (n=338), FIBRA. Campinas-SP, 2008-2009.

\begin{tabular}{|c|c|c|c|c|c|c|}
\hline \multirow[b]{2}{*}{ Variáveis } & \multicolumn{3}{|c|}{ Cuidadores } & \multicolumn{3}{|c|}{ Não cuidadores } \\
\hline & $\begin{array}{c}\text { Masculino } \\
(\mathrm{n}=116)\end{array}$ & $\begin{array}{c}\text { Feminino } \\
(\mathrm{n}=222)\end{array}$ & $\mathrm{p}$ & $\begin{array}{c}\text { Masculino } \\
(\mathrm{n}=116)\end{array}$ & $\begin{array}{c}\text { Feminino } \\
(\mathrm{n}=222)\end{array}$ & $\mathrm{p}$ \\
\hline \multicolumn{7}{|l|}{ Número de doenças } \\
\hline Zero & $\underline{23(19,8 \%)}$ & $17(07,7 \%)$ & $<0,001^{a}$ & $\underline{20(17,2 \%)}$ & $16(07,2 \%)$ & $0,002^{\mathrm{c}}$ \\
\hline 1 ou 2 & $\underline{62(53,5 \%)}$ & $90(40,5 \%)$ & & $\underline{61(52,6 \%)}$ & $105(47,3 \%)$ & \\
\hline 3 ou mais & $31(26,7 \%)$ & $\underline{115(51,8 \%)}$ & & $35(30,2 \%)$ & $\underline{101(45,5 \%)}$ & \\
\hline \multicolumn{7}{|l|}{ Fadiga } \\
\hline Não & $91(78,4 \%)$ & $158(71,2 \%)$ & 0,184 & $99 \underline{(85,3 \%)}$ & $165(74,3 \%)$ & $0,014^{\mathrm{d}}$ \\
\hline $\operatorname{Sim}$ & $24(20,7 \%)$ & $60(27,0 \%)$ & & $15(12,9 \%)$ & $\underline{54(24,3 \%)}$ & \\
\hline Não responderam & $1(0,9 \%)$ & $4(01,8 \%)$ & & $2(1,8 \%)$ & $3(1,4 \%)$ & \\
\hline \multicolumn{7}{|l|}{ Insônia } \\
\hline Não & $54(46,6 \%)$ & $81(36,5 \%)$ & 0,073 & $60(51,7 \%)$ & $107(48,2 \%)$ & 0,538 \\
\hline $\operatorname{Sim}$ & $62(53,4 \%)$ & $141(63,5 \%)$ & & $56(48,8 \%)$ & $115(51,8 \%)$ & \\
\hline \multicolumn{7}{|l|}{ Sintomas depressivos } \\
\hline Não (<5) & $94(81,0 \%)$ & $176(79,3 \%)$ & 0,760 & $99(85,3 \%)$ & $170(76,6 \%)$ & 0,058 \\
\hline $\operatorname{Sim}(\geq 6)$ & $22(19,0 \%)$ & $45(20,3 \%)$ & & $17(14,7 \%)$ & $52(23,4 \%)$ & \\
\hline Não responderam & $0(0,0 \%)$ & $1(0,4 \%)$ & & & & \\
\hline \multicolumn{7}{|l|}{ Satisfação com a vida } \\
\hline Menor $(\leq 21)$ & $63(54,3 \%)$ & $123(55,4 \%)$ & 0,943 & $59(50,8 \%)$ & $107(48,2 \%)$ & 0,918 \\
\hline Maior $(\geq 22)$ & $50(43,1 \%)$ & $96(43,2 \%)$ & & $56(48,3 \%)$ & $104(46,8 \%)$ & \\
\hline Não responderam & $3(2,6 \%)$ & $3(01,4 \%)$ & & $01(0,9 \%)$ & $11(5,0 \%)$ & \\
\hline \multicolumn{7}{|l|}{ AAVDs abandonadas } \\
\hline$<2$ & $15(12,9 \%)$ & $\underline{56(25,2 \%)}$ & $0,009^{\mathrm{b}}$ & $20(17,2 \%)$ & $\underline{75(33,8 \%)}$ & $0,005^{\mathrm{e}}$ \\
\hline $2-3$ & $56(48,3 \%)$ & $108(48,7 \%)$ & & $55(47,4 \%)$ & $88(39,6 \%)$ & \\
\hline$>3$ & $\underline{45(38,8 \%)}$ & $58(26,1 \%)$ & & $\underline{41(35,4 \%)}$ & $59(26,6 \%)$ & \\
\hline \multicolumn{7}{|l|}{ AIVDs } \\
\hline Independente (7) & $50(56,9 \%)$ & $92(58,6 \%)$ & 0,769 & $46(39,6 \%)$ & $71(32,0 \%)$ & 0,143 \\
\hline Dependência $(\leq 6)$ & $66(43,1 \%)$ & $130(41,4 \%)$ & & $69(59,5 \%)$ & $151(68,0 \%)$ & \\
\hline Não responderam & $0(0,0 \%)$ & $0(0,0 \%)$ & & $01(0,9 \%)$ & $0(0,0 \%)$ & \\
\hline \multicolumn{7}{|l|}{ Suporte Social } \\
\hline Baixo $(\leq 16)$ & $41(35,3 \%)$ & $67(30,2 \%)$ & 0,325 & $42(36,2 \%)$ & $73(32,9 \%)$ & 0,862 \\
\hline Médio (17-19) & $33(28,5 \%)$ & $53(23,9 \%)$ & & $31(26,7 \%)$ & $63(28,4 \%)$ & \\
\hline Alto $(\geq 20)$ & $42(36,2 \%)$ & $97(43,7 \%)$ & & $43(37,1 \%)$ & $82(36,9 \%)$ & \\
\hline Não responderam & $0(0,0 \%)$ & $5(02,2 \%)$ & & $0(0,0 \%)$ & $4(01,8 \%)$ & \\
\hline
\end{tabular}

${ }^{a} \operatorname{Sign}$ para $\mathrm{p}<0,001(\chi 2=23,45 ; \mathrm{GL}=2) ;{ }^{\mathrm{b}} \operatorname{Sign}$ para $\mathrm{p}=0,009(\chi 2=9,50 ; \mathrm{GL}=2) ;{ }^{\mathrm{c}} \operatorname{Sign}$ para $\mathrm{p}=0,002\left(\chi^{2}=12,08 ; \mathrm{GL}=2\right)$; ${ }^{\mathrm{S}} \operatorname{Sign}$ para $\mathrm{p}=0,014$ $(\chi 2=6,04 ; \mathrm{GL}=0,014) ;{ }^{\mathrm{e}}$ Sign para $\mathrm{p}=0,005\left(\chi^{2}=10,49 ; \mathrm{GL}=2\right)$.

AAVDs=Atividades Avançadas de Vida Diária; AIVDs=Atividades Instrumentais de Vida Diária. 
As comparações intragrupos de cuidadores e não cuidadores revelaram que somente no grupo de cuidadores foi observada uma frequência significantemente mais alta de dependência parcial para AIVD entre os idosos de 75-79 e 80 anos e mais, e frequência significativamente mais alta de idosos com independência total, nos grupos de 65-69 e 75-74 anos. Não foram observadas diferenças significativas nas outras variáveis no grupo de cuidadores quanto às frequências percentuais de idosos nas outras variáveis. No grupo de não cuidadores, não foram observadas quaisquer diferenças estatisticamente significativas (tabela 3). 


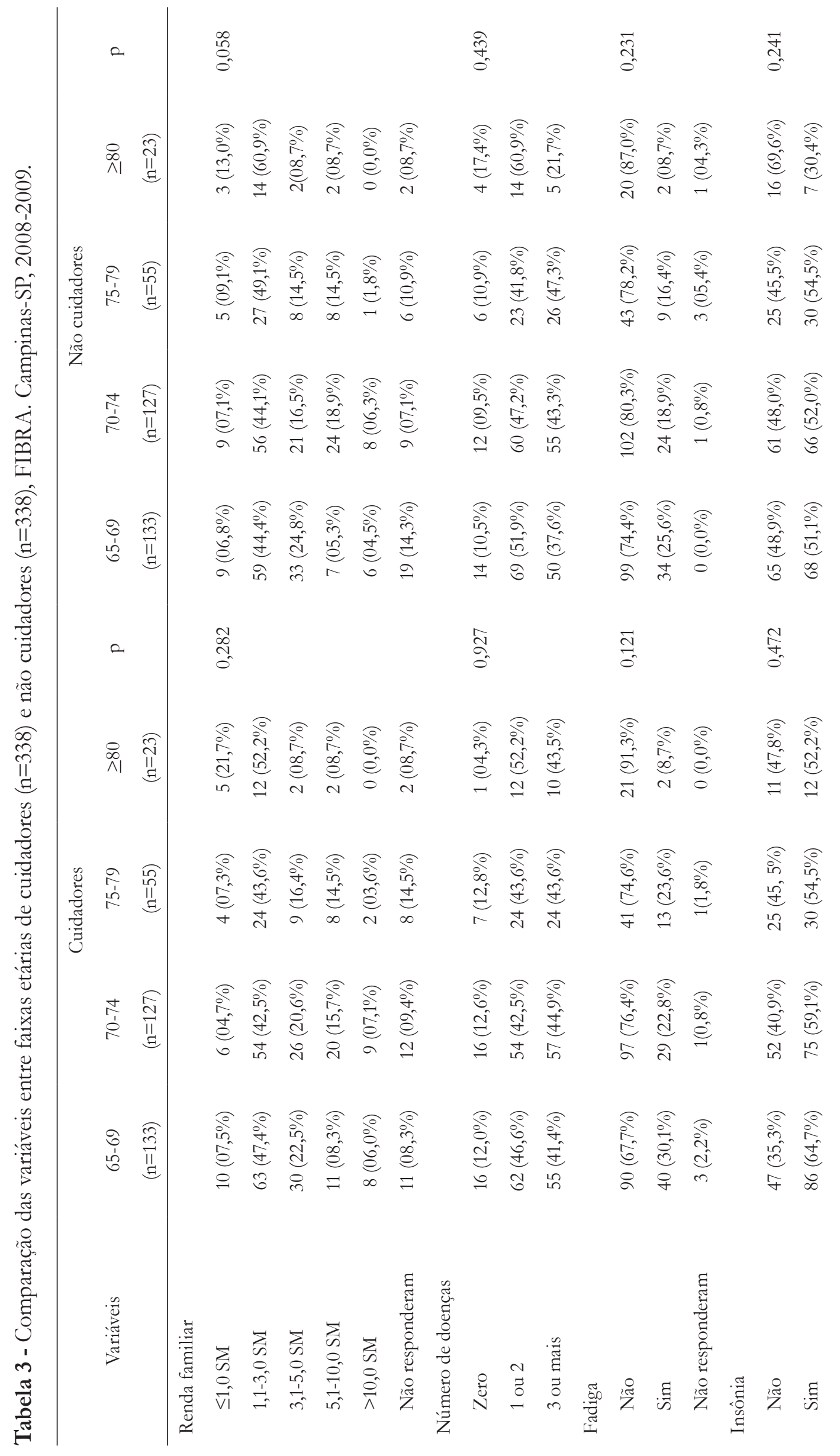




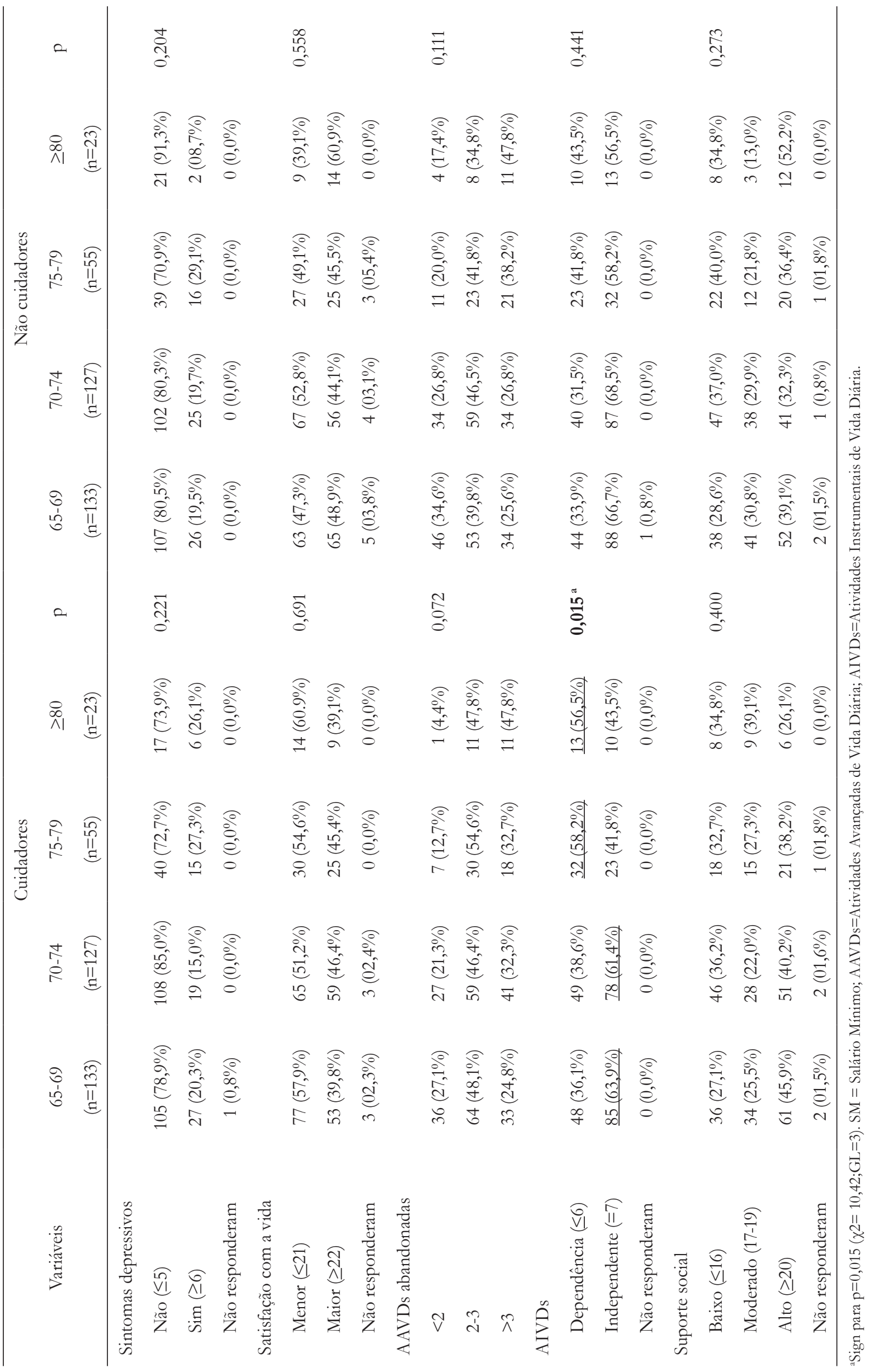


Entre os cuidadores, houve uma frequência significativamente mais alta de idosos que responderam negativamente aos itens de insônia entre aqueles com renda superior a dez salários mínimos do que com a renda de três a cinco salários mínimos. Igualmente entre os cuidadores com até três salários mínimos, foi encontrada frequência mais alta de sintomas depressivos do que entre aqueles com cinco salários mínimos ou mais. Em contrapartida, entre os cuidadores com maior rendimento familiar ( $\geq 3,1$ salários mínimos), houve a maior frequência de idosos independentes para AIVD. No grupo de não cuidadores, foi relatado um número significantemente maior de casos de insônia e de dependência em AIVD entre os mais pobres (renda de $\leq 1$ a 3 salários mínimos) do que entre os idosos com ganhos superiores a três salários mínimos (tabela 4). 


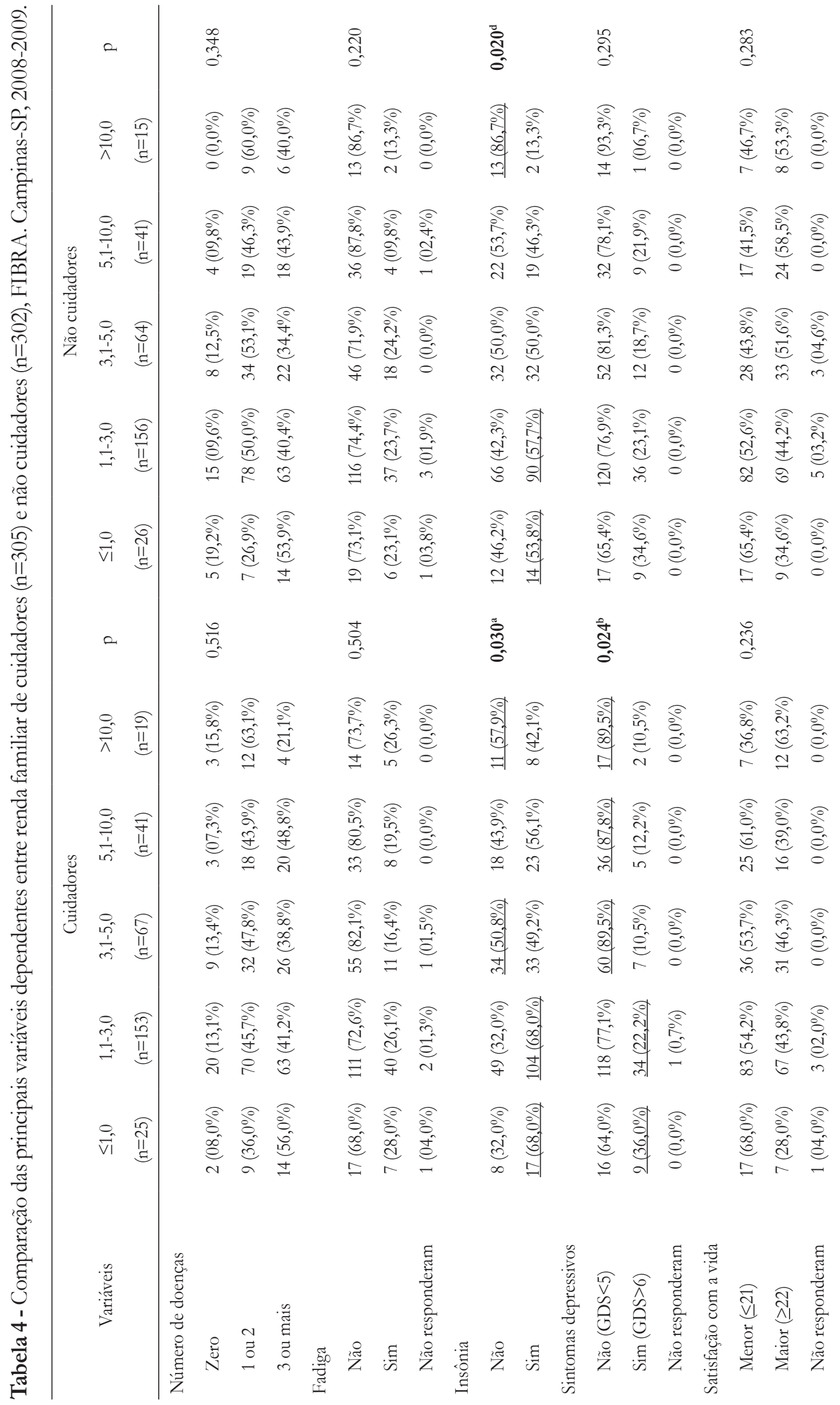




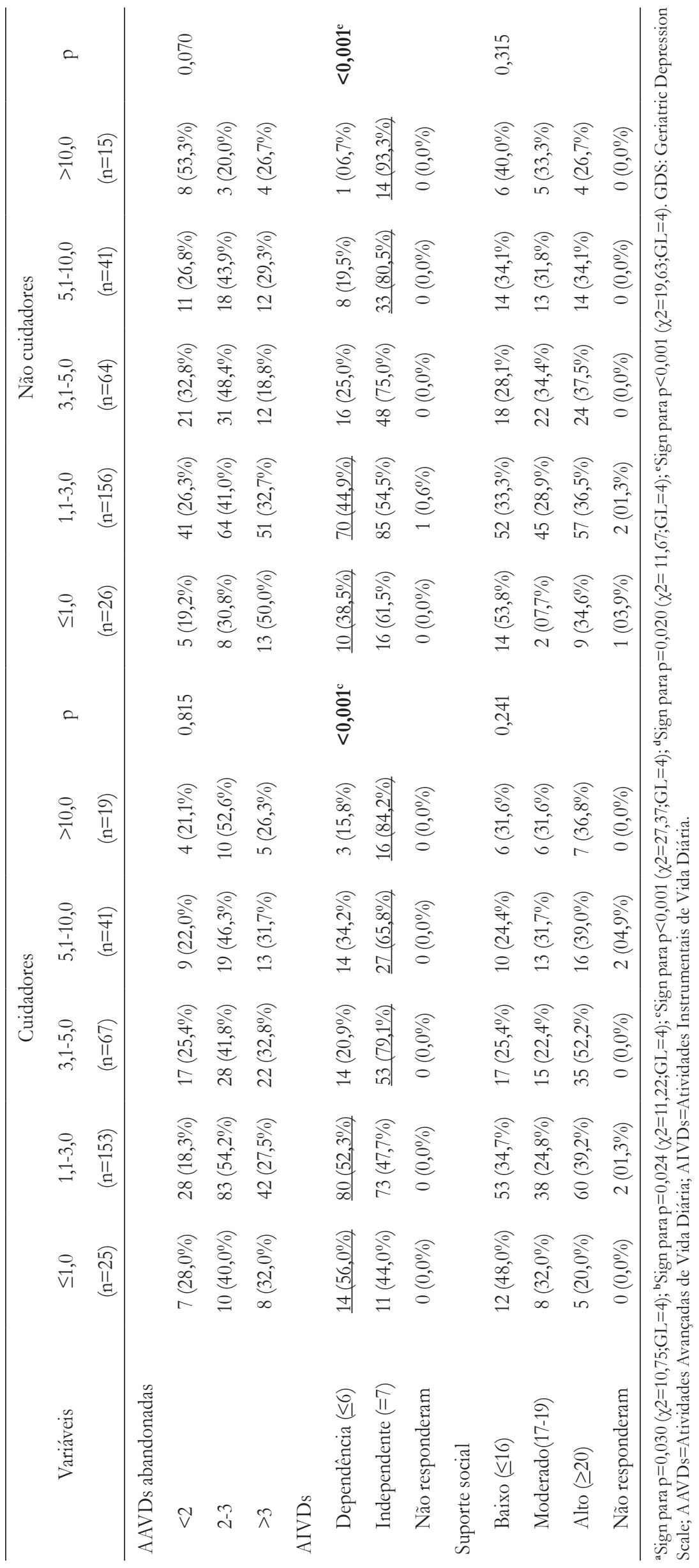




\section{DISCUSSÃO}

Os idosos cuidadores corresponderam a $29,7 \%$ da amostra. Prestar cuidado apareceu como uma condição mais frequente entre as mulheres, entre os idosos de 65 e 74 anos e entre os de menor renda. Verificou-se um percentual significativamente maior de queixas de insônia entre cuidadores do que não cuidadores. Entre os cuidadores e não cuidadores, baixa renda relacionou-se com maior frequência de relatos de insônia e de dependência parcial em AIVD, assim como relacionou-se com mais sintomas depressivos entre os cuidadores. Tanto entre cuidadores quanto não cuidadores, houve um percentual significativamente mais alto de mulheres do que de homens com três ou mais doenças e uma proporção maior de homens do que de mulheres que relataram diminuição no seu envolvimento social, assim como as mulheres não cuidadoras relataram mais fadiga do que os homens não cuidadores. Os cuidadores de 75 anos ou mais declararam mais limitação em atividades instrumentais de vida diária.

Muito embora a presente investigação não seja de natureza epidemiológica, a frequência de cuidadores foi comparável à encontrada em estudos populacionais brasileiros. No estudo de BambuíMG, a frequência foi de $23,7 \%,{ }^{23}$ e no SABE-SP, de $23,0 \%{ }^{24}$ Entretanto, houve discrepância em relação aos dados de estudos epidemiológicos internacionais - nos Estados Unidos foram estimados percentuais menores, $16 \%{ }^{25}$ Pinquart \& Sörensen ${ }^{26}$ relataram variação na prevalência de prestação do cuidado em diferentes populações e as atribuíram a diferenças étnicas, desigualdade socioeconômica, pobreza, piores condições de vida e arranjos de moradia multigeracionais.

À semelhança da maioria dos estudos sobre o cuidado, observou-se predominância de mulheres entre os cuidadores..$^{27} \mathrm{O}$ desempenho do cuidado é regulado por normas e valores sociais que determinam que as mulheres naturalmente cuidem de seus maridos, pais e sogros. ${ }^{3}$ Como em outros estudos, a prestação de cuidados foi mais comum entre os idosos mais jovens, de 65 a 79 anos, ${ }^{7,25}$ por um lado refletindo um efeito coorte e, por outro, o fato de eles terem mais energia do que os idosos mais velhos. No entanto, prevê-se no médio prazo, no Brasil, aumento na probabilidade de idosos muito idosos serem cuidados por outros idosos na mesma condição. ${ }^{1}$

Havia mais cuidadores do que não cuidadores com queixas de insônia, confirmando dados sobre a existência de pior qualidade do sono entre cuidadores, ${ }^{28}$ provavelmente derivada de pressões relacionadas ao cuidado. A vivência sob condições de escassez de recursos financeiros, porém, pode ter contribuído, uma vez que também se observou no presente estudo que os idosos mais pobres, de ambos os grupos, declararam sofrer de insônia, como verificado por Nomura et al. ${ }^{29}$ Adicionalmente, tanto os idosos cuidadores como os não cuidadores de baixa renda relataram pior desempenho funcional em AIVD, dados similares ao estudo de Gjonça et al. ${ }^{30}$ Pobreza, grande desigualdade socioeconômica e insuficiência de suporte institucional podem promover desvantagens sociais, gerar mais períodos de estresses crônicos durante toda vida e afetar o desenvolvimento físico e cognitivo dos envolvidos. ${ }^{31}$ Os idosos cuidadores tendem a ser mais prejudicados do que os não cuidadores.

Estudos com cuidadores apontaram associação entre baixa renda e presença de sintomas depressivos e maior número de doenças, ${ }^{5}$ assim como baixo nível de satisfação com a vida, especialmente entre aqueles com suporte social percebido como insuficiente. ${ }^{6}$ Esses dados são compatíveis com os resultados deste trabalho quanto aos sintomas depressivos, de modo especial entre os cuidadores mais pobres, sugerindo que prestar cuidados pode ser mais oneroso para eles, a ponto de prejudicar seu bem-estar subjetivo. Esses dados divergem dos de outras pesquisas sobre cuidado, ${ }^{4,5}$ que relatam maior número de doenças, maior grau de incapacidade e menor satisfação entre os cuidadores do que entre não cuidadores, de todos os grupos de renda. Uma possível explicação é que tanto cuidadores quanto não cuidadores avaliaram positivamente o suporte social que dispunham, expresso pelos altos escores de 
suporte social percebido, independentemente da renda. Este fato pode ter atenuado os efeitos dos riscos associados ao envelhecimento e à prestação de cuidados, como sugerido por Lincoln. ${ }^{32}$

A presença de mais doenças crônicas e fadiga entre as mulheres é um aspecto conhecido e bem explorado na literatura sobre cuidadores e na literatura gerontológica, ${ }^{4,33}$ assim como a tendência de maior privação de contatos e de atividades sociais entre os homens. ${ }^{3,34}$ É comum relacionar a prestação de cuidados à presença de maior número de doenças entre as mulheres. No entanto, é possível que as variáveis números de doença e fadiga sejam mais relacionadas a ser do sexo feminino do que prestar cuidados: neste estudo, as cuidadoras e as não cuidadoras relataram ter mais doenças do que os homens cuidadores e não cuidadores. Igualmente, as mulheres não cuidadoras relataram estar mais fatigadas do que os homens não cuidadores. Ser do sexo masculino parece ser igualmente uma explicação para a tendência dos homens cuidadores e não cuidadores a maior afastamento social do que aquele encontrado entre as mulheres cuidadoras e não cuidadoras. $O$ efeito de pertencimento ao gênero masculino estaria ligado ao fato de que os homens têm menos oportunidades para o desenvolvimento de habilidades sociais, emocionais e de cuidado ${ }^{3}$ do que as mulheres ao longo do curso de vida, o que resultaria em maior tendência a isolamento e de percepção de suporte social insuficiente na velhice, principalmente em situação de cuidado. Além disso, acredita-se que as mulheres são mais flexíveis, ao passo que os homens têm maior resistência em participar de atividades sociais que não sejam de seu interesse. ${ }^{34}$ Mesmo considerando a ausência de diferenças entre os homens e mulheres nesta investigação, é necessário admitir que é mais difícil cuidar com doenças e sob condição de isolamento social do que sob boas condições de saúde e envolvimento social.

As análises revelaram uma repercussão significativa da idade sobre o desempenho de AIVD em cuidadores, como relatado por Fredman et al..$^{35}$ No entanto, diferentemente da literatura, ${ }^{5}$ não houve significância estatística nas diferenças entre as condições de saúde, psicossociais e envolvimento social observadas nos grupos de cuidadores e de não cuidadores. Pinquart \& Sörenson ${ }^{5}$ verificaram piores condições de saúde entre cuidadores de idade mais avançada que interpretaram como produto a presença de distúrbios de comportamento entre os entes cuidados, o maior comprometimento e dificuldade para cuidar. Um dos fatores que poderá ter amenizado o ônus do cuidado para o grupo de cuidadores mais velhos terá sido a presença de ajudas provenientes das redes de relações informais. Outro fator a considerar é o alto nível de satisfação com a vida observado neste grupo, que possivelmente funcionou como variável protetora., ${ }^{4,33}$ Também podem ser indicadas mudanças socioemocionais e motivacionais adaptativas inerentes ao processo de envelhecimento, que permitem melhor ajustamento do self e preservação das reservas físicas, emocionais e cognitivas relevantes à adaptação as novas exigências contextuais, mais marcantes em cuidadores mais velhos. ${ }^{36}$

Limitações decorrentes do delineamento do estudo contraindicam fazer amplas generalizações com base nos dados observados. Não foram coletados dados sobre a duração, o diagnóstico e o prognóstico das doenças dos idosos que eram alvo dos cuidados. Tampouco estavam disponíveis dados sobre a eventual morte ou melhora da condição de saúde desses idosos, nem há quanto tempo teriam ocorrido esses eventos, determinando a cessação da exposição dos cuidadores ao ônus desse papel. Não havia dados sobre a qualidade das relações familiares, nem sobre ajuda instrumental disponível aos cuidadores. Além disso, o instrumento perguntava sobre o desempenho do cuidado nos últimos cinco anos, período possivelmente longo e sujeito a muitas intercorrências.

As respostas baseadas em autorrelato dependeram exclusivamente da subjetividade e da memória dos entrevistados. Podem ter ocorrido diferentes enfoques temporais e comparativos entre os cuidadores, que terão influenciado os dados, sem que os entrevistadores tivessem acesso aos elementos que controlaram as respostas. 
Mesmo considerando as variáveis "sexo" e "idade" nas análises intragrupos de idosos cuidadores, os resultados não podem ser atribuídos independentemente nem ao envelhecimento, nem ao cuidado. Novos estudos descritivos, de natureza longitudinal, com medidas de saúde e psicológicas mais discriminativas poderão oferecer respostas mais satisfatórias.

\section{CONCLUSÃO}

A despeito das limitações apontadas, os dados sugerem a necessidade de maior atenção médica e social aos idosos cuidadores, principalmente quando exibem piores condições de saúde física e psicológica, pior desempenho funcional, e participação social aliados a níveis baixos de renda.

Fazem-se necessários investimentos públicos e privados que assegurem trajetórias de melhores condições econômicas, de boa saúde, de bem-

\section{REFERÊNCIAS}

1. Camarano AA, Kanso S. Como as famílias brasileiras estão lidando com idosos que demandam cuidados e quais as perspectivas futuras? A visão mostrada pelas PNADs. In: Camarano AA, organizadora. Cuidados de longa duração para a população idosa: um novo risco social a ser assumido?. Rio de Janeiro: Instituto de Pesquisa Econômica Aplicada; 2010. p. 93-122.

2. Vitaliano P, Echeverria D, Shelkey M, Zhang J, Scanlan J. A cognitive psychophysiological model to predict functional decline in chronically stressed older adults. J Clin Psychol Med Settings 2007;14(3):177-90.

3. Giacomin KC, Uchoa E, Lima-Costa MF. Projeto Bambuí: a experiência do cuidado domiciliário por esposas de idosos dependentes. Cad Saude Publica 2005;21(5):1509-18.

4. Pinquart M, Sörensen S. Gender differences in caregiver stressors, social resources, and health: an updated meta-analysis. J Gerontol B Psychol Sci Soc Sci 2006;61(1):P33-45.

5. Pinquart M, Sörensen S. Correlates of physical health of informal caregivers: a meta-analysis. J Gerontol B Psychol Sci Soc Sci 2007;62(2):P126-37.

6. Borg C, Hallberg IR. Life satisfaction among informal caregivers in comparison with non-caregivers. Scand J Caring Sci 2006;20(4):427-38. estar psicológico à população de modo geral, de modo que os idosos possam ser favorecidos por cuidados de boa qualidade. A atenção econômica, social, educacional e psicológica às famílias com idosos deve ser priorizada considerando-se o papel central que desempenham no atendimento das necessidades dos mais velhos.

A busca de conhecimento sobre o cuidado e seus efeitos sobre a qualidade de vida dos idosos cuidadores e daqueles que são alvo do cuidado deverá favorecer a construção de modelos e teorias compatíveis com a realidade brasileira, em benefício da população que envelhece.

\section{AGRADECIMENTOS}

Aos colaboradores L.F. Araújo; G.A. Santos; A.C.G. Fortes-Burgos; E.P. Mantovani; F.S. Arbex Borim e S.V.S. Torres, pelo procedimento de coleta de dados.
7. Pinquart M, Sörensen S. Spouses, adult children, and children-in-law as caregivers of older adults: a metaanalytic comparison. Psychol Aging 2011;26(1):1-14.

8. Carter JH, Lyons KS, Stewart BJ, Archbold PG, Scobee R. Does age make a difference in caregiver strain? Comparison of young versus older caregivers in early-stage Parkinson's disease. Mov Disord 2010;25(6):724-30.

9. Smith SD, Gignac MA, Richardson D, Cameron JI. Differences in the experiences and support needs of family caregivers to stroke survivors: does age matter? Top Stroke Rehabil 2008;15(6):593-601.

10. Pinquart M, Sörenson S. Associations of stressors and uplifts of caregiving with caregiver burden and depressive mood: a meta-analysis. J Gerontol Psychol Scienc 2003;58B(2):P112-28.

11. Pearlin LI, Mullan JT, Semple SJ, Skaff MM. Caregiving and the stress process: an overview of concepts and their measures. Gerontologist 1990;30(5):583-94.

12. Neri AL, Yassuda MS, Araújo LF, Eulálio MC, Cabral BE, Siqueira MEC, et al. Metodologia e perfil sociodemográfico, cognitivo e de fragilidade de idosos comunitários de sete cidades brasileiras: estudo FIBRA. Cad Saúde Pública 2013;29(4):778-92. 
13. Brucki SMD, Nitrini R, Caramelli P, Bertolucci PHF, Okamoto IH. Sugestões para o uso do mini-exame do estado mental no Brasil . Arq Neuro-Psiquiatr 2003;61(3B):777-81.

14. Fortes-Burgos ACG, Neri AL, Cupertino APFB. Eventos estressantes, estratégias de enfrentamento, auto-eficácia e sintomas depressivos entre idosos residents na comunidade. Psicol Reflex Crítica 2008;21(1):74-82.

15. Batistoni SST, Neri AL, Cupertino APFB. Validade da escala de depressão do Center for Epidemiological Studies entre idosos brasileiros. Rev Saúde Pública 2007;41(4):598-605.

16. Almeida OP, Almeida SA. Short versions of the geriatric depression scale: a study of their validity for the diagnosis of a major depressive episode according to ICD-10 and DSM-IV. Int J Geriatr Psychiatry 1999;14(10):858-65.

17. Teixeira-Salmela LF, Magalhães Lde C, Souza AC, Lima Mde C, Lima RC, Goulart F. Adaptation of the Nottingham Health Profile: a simple measure to assess quality of life. Cad Saúde Pública 2004;20(4):905-14.

18. Neri AL. Bienestar subjetivo en la vida adulta y en la vejez: rumbo a una psicologia positiva en América Latina. Rev Latinoamericana Psicologia 2002;43(12):55-74.

19. Strawbridge WJ, Wallhagen MI, Cohen RD. Successful aging and well-being: self-rated compared with Rowe and Kahn. Gerontologist 2002;42(6):727-33.

20. Reuben DB, Laliberte L, Hiris J, Mor V. A hierarchical exercise scale to measure function at the Advanced Activities of Daily Living (AADL) level. J Am Geriatr Soc 1990;38(8):855-61.

21. Lawton MP, Brody EM. Assessment of older people: self-maintaining and instrumental activities of daily living. Gerontologist 1969;9(3):179-86.

22. Martire LM, Schulz R, Mittelmark MB, Newsom JT. Stability and change in older adults' social contact and social support: the Cardiovascular Health Study. J Gerontol B Psychol Sci Soc Sci 1999;54(5):S302-11.

23. Giacomin KC, Peixoto SV, Uchoa E, Lima-Costa MF. A population-based study on factors associated with functional disability among older adults in the Great Metropolitan Belo Horizonte, Minas Gerais State, Brazil. Cad Saude Publica 2008;24(6):1260-70.
24. Lebrão ML, Laurenti R. Saúde, bem-estar e envelhecimento: o estudo SABE no Município de São Paulo. Rev Bras Epidemiol 2005;8(2):127-41.

25. Dahlberg L, Demack S, Bambra C. Age and gender of informal carers: a population-based study in the UK. Health Soc Care Community 2007;15(5):439-45.

26. Pinquart M, Sörensen S. Ethnic differences in stressors, resources, and psychological outcomes of family caregiving: a meta-analysis. Gerontologist 2005;45(1):90-106.

27. Roberto KA, Jarrott SE. Family caregivers of older adults: a life span perspective. Fam Relat 2008;57(1):100-11.

28. Arber S, Bote M, Meadows R. Gender and socioeconomic patterning of self-reported sleep problems in Britain. Soc Sci Med 2009;68(2):281-9.

29. Nomura K, Yamaoka K, Nakao M, Yano E. Social determinants of self-reported sleep problems in South Korea and Taiwan. J Psychosom Res 2010;69(5):435-40.

30. Gjonça E, Tabassum F, Breeze E. Socioeconomic differences in physical disability at older age. J Epidemiol Community Health 2009;63(11):928-35.

31. Kahn JR, Pearlin LI. Financial strain over the life course and health among older adults. J Health Soc Behav 2006;47(1):17-31.

32. Lincoln KD. Financial strain, negative interactions, and mastery: pathways to mental health among older African Americans. J Black Psychol 2007;33(4):439-62.

33. Kim IH. Age and gender differences in the relation of chronic diseases to activity of daily living (ADL) disability for elderly South Koreans: based on representative data. J Prev Med Public Health 2011;44(1):32-40.

34. Davidson K, Daly T, Arber S. Older men, social integration and organisational activities. Soc Policy Society 2003;2(2):81-9.

35. Fredman L, Doros G, Ensrud KE, Hochberg MC, Cauley JA. Caregiving intensity and change in physical functioning over a 2-year period: results of the caregiver-study of osteoporotic fractures. Am J Epidemiol 2009;170(2):203-10.

36. Scheibe S, Carstensen LL. Emotional aging: recent findings and future trends. J Gerontol B Psychol Sci Soc Sci 2010;65B(2):135-44. 